\title{
PARENTAL PERCEPTION OF THE CHILDHHOOD IMMUNISATION PROGRAMME IN IRELAND
}

L. Morrissey ${ }^{1}$, S. Musleh ${ }^{1}$, R. Stewart ${ }^{1}$, A. Feeney ${ }^{1}$, Z. Muravec ${ }^{1}$, J. Meehan ${ }^{2}$, A.M. Murphy ${ }^{2}$, E. Roche ${ }^{2}$

Department of Paediatrics, Trinity College Dublin, Dublin, Ireland

Background and aims: Ireland introduced the first National Childhood Immunisation Programme in 1949 with the BCG. The most recent revision occurred in 2010 with the addition of the HPV vaccine. Vaccination uptake in Ireland is high.The majority of Irish children are fully immunised. Our aim was to investigate parental experience with the immunisation programme and to explore whether previously identified objections and concerns regarding safety still exist.

Methods: We randomly distributed a specifically constructed self-report survey to 200 parents at a Children's Hospital in Dublin during March 2011. Questions explored demographics, previous experiences and opinions regarding childhood vaccination. Data was analysed using SPSS.

Results: Of the 200 participants, $94 \%(\mathrm{n}=188)$ reported satisfaction with information they received regarding immunisation prior to deciding to immunise and $92 \%(n=184)$ stated that their child's vaccinations were fully up to date. However, $45 \%$ of those surveyed reported concerns about potential side effects. Overall, $82 \%$ of parents reported a 'very positive' or a 'positive' experience regarding vaccination. Only $1 \%$ of parents reported a previous negative experience to vaccination. Interestingly, $52 \%$ of parents reported that their child received the $\mathrm{H} 1 \mathrm{~N} 1$ vaccine. Previous negative experiences with vaccination, dissatisfaction with information and concern about side-effects were the reasons cited by those who had not had the children fully immunised.

Conclusion: A significant proportion of parents surveyed expressed concerns regarding side effects of immunisation, however most follow the recommended schedule. Our study suggests that Irish parents are satisfied with the immunisation programme and overall most have had a positive experience. 